\title{
Pareto utility
}

\author{
Masako Ikefuji • Roger J. A. Laeven • \\ Jan R. Magnus • Chris Muris
}

Received: 2 September 2011 / Accepted: 3 January 2012 / Published online: 26 January 2012

(C) The Author(s) 2012. This article is published with open access at Springerlink.com

\begin{abstract}
In searching for an appropriate utility function in the expected utility framework, we formulate four properties that we want the utility function to satisfy. We conduct a search for such a function, and we identify Pareto utility as a function satisfying all four desired properties. Pareto utility is a flexible yet simple and parsimonious two-parameter family. It exhibits decreasing absolute risk aversion and increasing but bounded relative risk aversion. It is applicable irrespective of the probability distribution relevant to the prospect to be evaluated. Pareto utility is therefore particularly suited for catastrophic risk analysis. A new and related class of generalized exponential (gexpo) utility functions is also studied. This class is particularly relevant in situations where absolute risk tolerance is thought to be concave rather than linear.
\end{abstract}

Keywords Parametric utility - Hyperbolic absolute risk aversion (HARA) · Exponential utility $\cdot$ Power utility

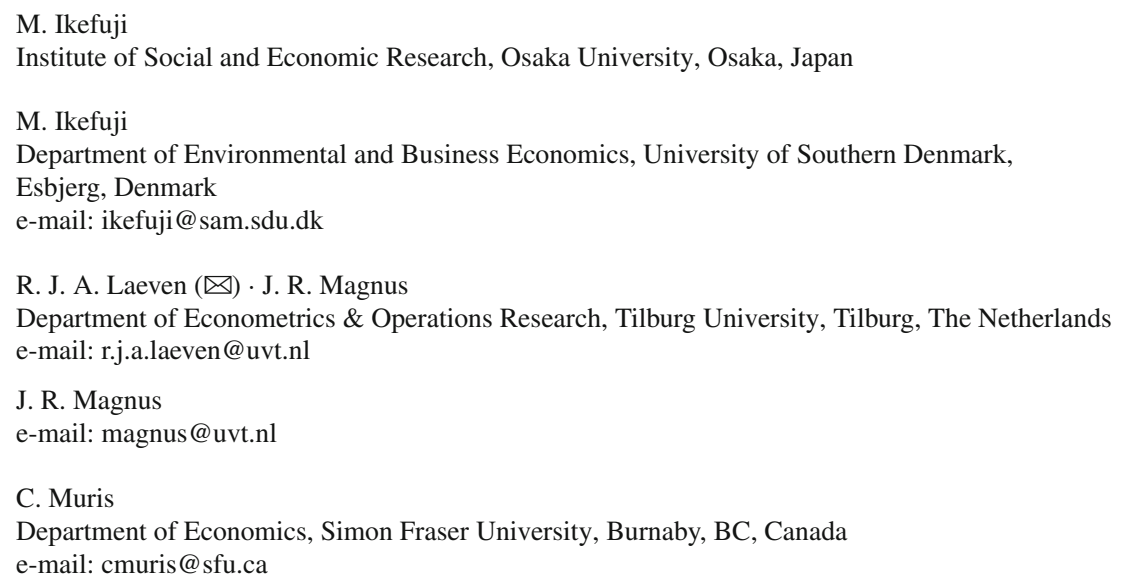




\section{JEL Classification D81}

\section{Introduction}

In many decision theories, including expected utility and the most common nonexpected utility theories, the utility function $U$ is unique up to positive affine transformations, that is, $U$ is a cardinal (interval) scale. In searching for a suitable utility function, it is the curvature of the function that is of interest: it contains all information pertaining to a cardinal scale. To measure curvature we typically normalize the second derivative $U^{\prime \prime}$, which is not invariant to positive affine transformations in $U$, by dividing by the first derivative (de Finetti 1952; Pratt 1964; Yaari 1969; Arrow 1971). This gives the Arrow-Pratt measure of absolute risk aversion,

$$
\operatorname{ARA}(x)=\frac{-\mathrm{d} \log U^{\prime}(x)}{\mathrm{d} x}=\frac{-U^{\prime \prime}(x)}{U^{\prime}(x)}
$$

also referred to as the concavity index.

Up to positive affine transformations, there is precisely one function family with constant and positive absolute risk aversion (CARA), namely the cumulative distribution function of the exponential distribution,

$$
U(x)=1-\mathrm{e}^{-x / \lambda} \quad(\lambda>0) .
$$

Equally important is relative risk aversion,

$$
\operatorname{RRA}(x)=\frac{-\mathrm{d} \log U^{\prime}(x)}{\mathrm{d} \log x}=\frac{-x U^{\prime \prime}(x)}{U^{\prime}(x)} .
$$

Again, there exists precisely one function family with constant and positive relative risk aversion (CRRA), namely the power function,

$$
U(x)=\frac{x^{1-\alpha}-1}{1-\alpha} \quad(\alpha>0) .
$$

Throughout, unless stated otherwise, we consider only non-negative inputs $(x \geq 0)$. Exponential utility is bounded from above and below, and satisfies $\operatorname{ARA}(x)=1 / \lambda$ and $\operatorname{RRA}(x)=x / \lambda$. Thus, it exhibits constant ARA and increasing RRA. Power utility is either unbounded from above $(0<\alpha<1)$ or from below $(\alpha>1)$ or both $(\alpha=1)$, and satisfies $\operatorname{ARA}(x)=\alpha / x$ and $\operatorname{RRA}(x)=\alpha$. Thus, it exhibits decreasing ARA and constant RRA.

In both theory and applications, power and exponential utility - in this order-are the most commonly used parametric families of utility functions. Some theoretical advantages of the power and exponential families are listed in Castagnoli and LiCalzi (1996); see also Eeckhoudt and Gollier (1995, Section 4.3). If we are interested in inputs $x$ 'remote from' 0 , as is common in macroeconomics and finance, then power utility is often appropriate, at least for the purpose of fitting data; see Wakker (2008) 
and references therein. Exponential utility is less appropriate for such inputs, because its RRA increases without bound and therefore often provides a poor fit (cf., Eeckhoudt and Gollier 1995, p. 50). If, on the other hand, we are interested in near-catastrophe cases ('small' $x$ ), as in the insurance literature, then exponential utility is often used (Gerber 1979, Chapter 5). Exponential utility implies $\operatorname{ARA}(0)=1 / \lambda<\infty$ and thus avoids the extreme behavior of power utility near $x=0$, with ARA exploding at a high rate when $x$ tends to 0 . Rabin (2000, p. 1287), noted on theoretical grounds that, under expected utility, power preferences should not be used when both 'large' and 'small' inputs are relevant. But, due to its unbounded RRA, the same conclusion is true for exponential preferences. If we are interested in the whole non-negative range of inputs, then more flexible families are required.

The purpose of this article is to find a family of utility functions with sufficient flexibility so that it can be applied on the whole range of non-negative inputs. In addition, we want our family of utility functions to be applicable irrespective of the (objective or subjective) probability distribution pertaining to the prospect to be evaluated. Unless stated otherwise we choose expected utility as our decision theory. In practice, the requirement of applicability irrespective of the prospect's probability distribution is particularly relevant when dealing with catastrophic risks. Such risks are typically modeled using heavy-tailed probability distributions, which may induce infinite expected (marginal) utility when the utility function is left unrestricted.

More specifically, we search for a utility function satisfying the following four (primarily normative) properties:

P1: ARA is non-increasing and convex, and RRA is non-decreasing and concave;

P2: Expected utility is finite irrespective of the probability distribution;

P3: Expected marginal utility is finite irrespective of the probability distribution;

P4: Utility behaves power-like for inputs remote from 0 : $\operatorname{RRA}(\infty)<\infty$.

Property P1 follows the spirit of Arrow (1971) on theoretical grounds; see also Eeckhoudt and Gollier (1995, Section 4.2, Hypotheses 4.1 and 4.2). Empirical justifications for increasing RRA for decision under risk are provided, inter alia, by Friend and Blume (1975), Binswanger (1980), Holt and Laury (2002), and Post et al. (2008).

Property P2 is satisfied if and only if utility is bounded from above and below. If utility is bounded, then we may assume, without loss of generality, that it is bounded between zero and one. Hence, $U$ behaves like a cumulative distribution function, and we shall utilize this feature in Section 4.

Property P3 is satisfied if and only if $\int_{0}^{\gamma} \operatorname{ARA}(x) \mathrm{d} x<\infty$ for some $\gamma>0$ (Ikefuji et al. 2011). Finally, property P4 is satisfied if and only if RRA $(x)$ remains finite as $x \rightarrow$ $\infty$. This is normatively appealing (Eeckhoudt and Gollier 1995, p. 50; Castagnoli and LiCalzi 1996) and empirically justified (Chiappori and Paiella 2008; Wakker 2008).

We achieve our purpose by identifying a family of utility functions which satisfies all properties $\mathrm{P} 1-\mathrm{P} 4$ :

$$
U(x)=1-(1+x / \lambda)^{-k} \quad(k>0, \lambda>0) .
$$

We call this function Pareto utility, because the translation $z=x+\lambda$ yields $U(z)=$ $1-(z / \lambda)^{-k}$, defined for $z \geq \lambda$, which is the cumulative distribution function of the Pareto distribution. Pareto utility is a flexible yet simple and parsimonious family of 
utility functions. Due to properties P2 and P3, it is particularly suited for heavy-tailed risk analysis.

The outline of this article is as follows. In Section 2, we analyze the class $\mathcal{U}$ of utility functions which satisfy property $\mathrm{P} 1$, that is, non-decreasing and convex ARA and non-increasing and concave RRA. Two subclasses suggest themselves. In Section 3, we study the HARA subclass. Pareto utility is identified as the unique member of the HARA subclass satisfying all four properties P1-P4. Section 4 connects Pareto utility to the Burr distribution, providing a further rationale for Pareto utility. In Section 5 we study another subclass of $\mathcal{U}$, leading to 'gexpo' utility, a generalization of exponential utility, which is of independent interest. In Section 6, we briefly discuss expo-power utility, an increasingly popular family of utility functions. Section 7 provides a graphical comparison of the exponential, power, Pareto, gexpo and expo-power families of utility functions. Section 8 concludes.

\section{The class $\mathcal{U}$}

In the spirit of Arrow (1971), we confine our search to those utility functions satisfying property $\mathrm{P} 1$. This is a rich class, which we shall call class $\mathcal{U}$.

Definition 1 Let $U(x)$ be defined for $x \geq 0$, twice differentiable, and such that $U^{\prime}(x)>0$ and $U^{\prime \prime}(x)<0$ for $x>0$. If $\operatorname{ARA}(x)$ is non-increasing and convex and if $\operatorname{RRA}(x)$ is non-decreasing and concave for all $x>0$, then we say that the function $U$ belongs to the class $\mathcal{U}$.

Many well-known families of utility functions will appear to be subclasses of $\mathcal{U}$. Both power and exponential utility are obviously subclasses of $\mathcal{U}$ (and hence satisfy P1). Exponential utility also satisfies P2 and P3, but not P4; while power utility satisfies $\mathrm{P} 4$, but not $\mathrm{P} 2$ and $\mathrm{P} 3$.

It will be useful to define absolute risk tolerance as

$$
T(x)=\frac{-U^{\prime}(x)}{U^{\prime \prime}(x)}=\frac{1}{\operatorname{ARA}(x)}=\frac{x}{\operatorname{RRA}(x)} .
$$

We have $T(x)=\lambda$ for exponential utility and $T(x)=x / \alpha$ for power utility, and hence both utility functions exhibit linear absolute risk tolerance. Utility functions with linear absolute risk tolerance are said to display 'hyperbolic absolute risk aversion' (HARA), and are particularly useful to derive analytical results (Gollier 2001, Section 2.6). Suppose $U$ is four times differentiable. Defining

$$
R_{1}(x)=\frac{x T^{\prime}(x)}{T(x)}, \quad R_{2}(x)=\frac{x^{2} T^{\prime \prime}(x)}{T(x)},
$$

and differentiating $\operatorname{ARA}(x)=1 / T(x)$ and $\operatorname{RRA}(x)=x / T(x)$ twice with respect to $x$, we find

$$
\begin{aligned}
& \operatorname{ARA}^{\prime}(x) \leq 0 \Longleftrightarrow R_{1}(x) \geq 0, \quad \operatorname{RRA}^{\prime}(x) \geq 0 \Longleftrightarrow R_{1}(x) \leq 1, \\
& \operatorname{ARA}^{\prime \prime}(x) \geq 0 \Longleftrightarrow R_{2}(x) \leq 2 R_{1}^{2}(x),
\end{aligned}
$$


and

$$
\operatorname{RRA}^{\prime \prime}(x) \leq 0 \Longleftrightarrow R_{2}(x) \geq-2 R_{1}(x)\left(1-R_{1}(x)\right), \quad x>0
$$

Thus we obtain the following result.

Proposition 1 Let $U(x)$ be defined for $x \geq 0$, four times differentiable, and such that $U^{\prime}(x)>0$ and $U^{\prime \prime}(x)<0$ for $x>0$. Then $U$ belongs to $\mathcal{U}$ if and only if, for $x>0$,

$$
0 \leq R_{1}(x) \leq 1, \quad-2 R_{1}(x)\left(1-R_{1}(x)\right) \leq R_{2}(x) \leq 2 R_{1}^{2}(x) .
$$

The function $R_{1}$ captures the third derivative of the utility function $U$. It represents the elasticity of absolute risk tolerance $\mathrm{d} \log T(x) / \mathrm{d} \log x$, which equals minus the elasticity of ARA and one minus the elasticity of RRA. Thus, with $0 \leq R_{1}(x) \leq 1$, a $1 \%$ increase in $x$ cannot lead to a more than $1 \%$ increase in $T$ or RRA, or a more than $1 \%$ decrease in ARA. The function $R_{2}$ captures the fourth derivative of $U$.

For power utility, we have $R_{1} \equiv 1$, for exponential utility we have $R_{1} \equiv 0$, and these two utility functions are therefore corner cases in $\mathcal{U}$. For both power and exponential utility we have $R_{2} \equiv 0$. The two cases $R_{2} \equiv 0$ and $R_{1} \equiv r(0 \leq r \leq 1)$ thus suggest themselves as natural extensions to power and exponential utility, and we analyze these two cases in Sections 3 and 5, respectively. Notice that the definition of these two classes of utility functions $\left(R_{2} \equiv 0\right.$ and $\left.R_{1} \equiv r, 0 \leq r \leq 1\right)$ has a similar flavor as, but is more general than, the definition of the exponential and power families ( $T \equiv \lambda$ and $x / T(x) \equiv \alpha$ or equivalently $R_{1} \equiv 0$ and $R_{1} \equiv 1$, respectively).

\section{The class $\boldsymbol{R}_{2} \equiv \mathbf{0 :}$ HARA utility}

The class $R_{2} \equiv 0$ is characterized by $T(x)=a x+b(x \geq 0)$, and hence contains all utility functions with non-negative inputs that display linear HARA; see Mossin (1968) and Merton (1971). Because we consider only non-negative inputs, we restrict attention to the corresponding restriction of the HARA class, without explicitly mentioning this henceforth when we use the designation 'HARA'. Since in this case $R_{1}(x)=a x /(a x+b)$, we find that $U$ belongs to $\mathcal{U}$ if and only if $a \geq 0, b \geq 0$, and $a+b>0$.

From (1) and (6), we find that the HARA class is also characterized by the differential equation

$$
\mathrm{d} \log U^{\prime}(x)+\frac{\mathrm{d} x}{a x+b}=0 \quad(a \geq 0, b \geq 0, a+b>0) .
$$

There are three cases. For $a=0$ and $b>0$, we find the CARA utility function (exponential); for $a>0$ and $b=0$, we find the CRRA utility function (power); and for $a>0$ and $b>0$, we find the utility function in two steps. We first obtain $U^{\prime}(x)=A(a x+b)^{-1 / a}$, and then, letting $\alpha=1 / a$ and $\lambda=b / a$,

$$
U(x)=\frac{(x+\lambda)^{1-\alpha}-1}{1-\alpha} \quad(\alpha>0, \lambda>0),
$$


apart from positive affine transformations. We see that

$$
\operatorname{ARA}(x)=\frac{\alpha}{x+\lambda}, \quad \operatorname{RRA}(x)=\frac{\alpha x}{x+\lambda}
$$

Both RRA and ARA are bounded from above and below in this case. When $0<\alpha \leq 1$ utility is bounded from below but unbounded from above; when $\alpha>1$ utility is bounded from above and below. Marginal utility is bounded from above and below for every $\alpha$, also at zero.

We conclude that the HARA class contains seven types of utility functions, as follows:

$U$ satisfies $\mathrm{P} 1$ and $\mathrm{P} 4$ (power):

$$
\begin{aligned}
& U(x)=\log x, \\
& U(x)=x^{r}(0<r<1), \\
& U(x)=1-x^{-k}(k>0) ;
\end{aligned}
$$

$U$ satisfies $\mathrm{P} 1, \mathrm{P} 3$, and $\mathrm{P} 4$ :

$$
U(x)=\log (1+x / \lambda)(\lambda>0),
$$$$
U(x)=(1+x / \lambda)^{r}-1(0<r<1, \lambda>0) \text {; }
$$

$U$ satisfies $\mathrm{P} 1, \mathrm{P} 2$, and $\mathrm{P} 3$ (exponential):

$$
U(x)=1-\mathrm{e}^{-x / \lambda}(\lambda>0)
$$

$U$ satisfies $\mathrm{P} 1-\mathrm{P} 4$ (Pareto):

$$
U(x)=1-(1+x / \lambda)^{-k}(k>0, \lambda>0),
$$

where we have normalized the functions-without loss of generality-such that if there is a lower bound, it is zero; and if there an upper bound, it is one.

All members of the HARA class belong to $\mathcal{U}$. If a member of the HARA class does not satisfy P3, then it belongs to the power family (and vice versa). If a member of the HARA class satisfies $\mathrm{P} 3$, then $\mathrm{RRA}(0)=0$ and $\mathrm{ARA}(0)<\infty$. The extreme behavior of the power family near $x=0$, where ARA explodes at a high rate, may generate important problems when inputs are not bounded away from 0 , because expected marginal utility and the expected pricing kernel may then be infinite, thus complicating expected utility analysis. Modifying the unit of inputs (to $\tilde{x}=a x, a>0$ ) does not affect the power family - an exclusive property of this family_but does not remedy these problems, because RRA(0) is still strictly positive, hence P3 is violated. Modifying the level of inputs (to $\tilde{x}=x+b$ ) could conceivably solve the problems, but does affect the power family. We note in passing that for all HARA utility functions satisfying P3, a modification of the unit of inputs can be nullified by adjusting the parameter $\lambda$. Also, within the HARA class, only the exponential family is invariant to a modification of the level of inputs.

The function (7) is well-known if we interpret $\lambda$ as initial wealth. But (7) can also be interpreted, more generally, as a two-parameter utility function (Harrison et al. 2007). It is an appealing, seemingly more appropriate, alternative to the power family (4), characterized by the two parameters $\alpha$ and $\lambda$. Intuitively, the parameter $\alpha$ describes the degree of relative risk aversion for large inputs $(\operatorname{RRA}(\infty)=\alpha)$, while $\alpha / \lambda$ describes the degree of absolute risk aversion for small inputs $(\operatorname{ARA}(0)=\alpha / \lambda)$. Thus, $\alpha$ may be calibrated to resemble power utility for inputs remote from 0 as is commonly found 
empirically; see property P4. Once $\alpha$ has been calibrated, the parameter $\lambda$ may, for example, be calibrated to resemble exponential utility for inputs near 0 .

We note that, if we allow $\lambda<0$, then the behavior of (7) is quite different, as is the case for Stone-Geary utility functions. The parameter $\lambda$ then plays the role of subsistence level. With $\lambda>0$, RRA $(0)=0$ and RRA is increasing, while with $\lambda<0$, $\operatorname{RRA}(-\lambda)=\infty$ and, for $x>-\lambda$, RRA is decreasing.

An additional problem with the power family is the extreme behavior of its derivatives at $x=0$. This implies that in a setting — not considered here-with both positive and negative inputs including $x=0$, the loss aversion index of Köbberling and Wakker (2005), defined as the ratio of one-sided derivatives at $x=0$, behaves improperly under power utility. In contrast, all HARA utility functions satisfying P3 have smooth derivatives at $x=0$, and allow for a generalization to a setting with both positive and negative inputs that induces proper behavior of this loss aversion index.

Only one of the seven utility functions in the HARA class satisfies all four properties P1-P4, namely the last one: Pareto utility. This is the special case of (7) where $\alpha>1$, that is, the only member of (7) which is bounded from above and below. In the next section, we shall arrive at the same utility function through a different route.

\section{Bounded utility}

In the previous section, we started with property $\mathrm{P} 1$, and then demonstrated that in the subclass $R_{2} \equiv 0$ there is precisely one utility function, the Pareto function, satisfying $\mathrm{P} 1-\mathrm{P} 4$. In this section, we start with property $\mathrm{P} 2$, bounded utility. If utility is bounded from above and from below, we may assume-without loss of generality - that the lower bound is zero and the upper bound one. This means that the utility function can be viewed as a cumulative distribution function. The question then becomes: what is the class of distribution functions that satisfies P1-P4?

We cannot answer this question completely, but we can get close to it by studying the Burr (or Singh-Maddala) distribution (Burr 1942; Burr and Cislak 1968), defined as

$$
U(x)=1-\left(1+(x / \lambda)^{c}\right)^{-k} \quad(k>0, \lambda>0, c>0) .
$$

This is a three-parameter family of distribution functions with the property that many of the known distribution functions are special or limiting cases. It is therefore an appropriate function to approximate an unknown distribution function. Absolute risk tolerance is given by

$$
T(x)=\frac{\lambda\left(1+(x / \lambda)^{c}\right)(x / \lambda)}{(c k+1)(x / \lambda)^{c}+(1-c)} .
$$

One verifies that Burr utility always satisfies P2 and P4. It satisfies P1 if and only if $c \leq 1$, and it satisfies P3 if and only if $c=1$. Hence, the only special case of (8) which satisfies P1-P4 is the family where $c=1$, and this turns out to be the Pareto utility function. In that case, $T(x)=(x+\lambda) /(k+1)$. 
Thus we have proved that both in the HARA class and in the Burr class, Pareto utility is the unique member satisfying properties P1-P4. These two facts make Pareto utility an interesting candidate when one has to choose the functional form of utility. It is simple and parsimonious, and it shares the computationally attractive properties of the HARA class. We have not been able to identify any other simple and parsimonious utility function satisfying all four properties.

The two parameters $k$ and $\lambda$ jointly characterize Pareto utility, and provide the flexibility to match power-like behavior for inputs remote from 0 through calibrating $k(\operatorname{RRA}(\infty)=k+1)$, while avoiding the rapidly exploding degrees of absolute risk aversion that the power function exhibits for inputs near 0 through calibrating $\lambda$, given $k$, to the desired degrees of absolute risk aversion for small inputs $(\operatorname{ARA}(0)=(k+1) / \lambda)$. Note in this respect that the parameter $\lambda$ is in general not initial wealth.

Pareto utility, like any member of the HARA class, has a completely monotone first derivative with higher-order derivatives of alternating sign. Its index of $n$ th-order risk attitude (Denuit and Eeckhoud 2010) is given, for $n \geq 2$, by

$$
(-1)^{n+1} \frac{U^{(n)}(x)}{U^{\prime}(x)}=[(1+k)(2+k) \cdots((n-1)+k)](x+\lambda)^{-n+1} .
$$

\section{The class $R_{1} \equiv r$ : gexpo utility}

Let us return to the class $\mathcal{U}$, that is the class of utility functions satisfying P1. Earlier we identified two intuitive subclasses, namely $R_{2} \equiv 0$ and $R_{1} \equiv r$. The former was studied in Section 3; the latter is studied in this section.

The class $R_{1} \equiv r(0 \leq r \leq 1)$ is characterized by $T(x)=x^{r} / \beta(\beta>0)$, and we see that $R_{2}(x)=-r(1-r)$. When $0 \leq r<1$, the utility function satisfies properties P1-P3, but not P4; when $r=1$, we have power utility satisfying properties $\mathrm{P} 1$ and $\mathrm{P} 4$, but not $\mathrm{P} 2$ and $\mathrm{P} 3$. Hence, no member in this class satisfies all four properties. Nevertheless, the case $0 \leq r \leq 1$ is clearly intermediate between exponential $(r=0)$ and power $(r=1)$, and therefore of interest.

To solve $U(x)$ from $T(x)$ we first obtain marginal utility $U^{\prime}(x)$ from

$$
\mathrm{d} \log U^{\prime}(x)+\beta x^{-r} \mathrm{~d} x=0 .
$$

This yields $U^{\prime}(x)=A \exp \left(-\beta x^{1-r} /(1-r)\right)$, where $A$ is an arbitrary positive constant. Reparameterize by letting $p=1 /(1-r)$ and $\lambda=(1 / \beta)^{1 /(1-r)}$, excluding henceforth the power family $(r=1)$, and choose $A$ such that $U^{\prime}(x)$ is a proper density function. Then,

$$
U^{\prime}(x)=\frac{p^{p} \mathrm{e}^{-p h(x)}}{\lambda \Gamma(p+1)}, \quad h(x)=(x / \lambda)^{1 / p} \quad(p \geq 1, \lambda>0)
$$

is a special case of the three-parameter generalized gamma density, other special cases of which are the two-parameter gamma, the Weibull, and the lognormal densities (Stacy 1962; Johnson et al. 1995). This density, first proposed by Subbotin (1923), 
is sometimes called the 'exponential power' or the 'power(ed) exponential' density (Box and Tiao 1973; Johnson et al. 1995, pp. 195-198); we shall call it the 'gexpo' density, because it generalizes the exponential density from $p=1$ to $p \geq 1$. Our gexpo class differs from the expo-power family of utility functions introduced by Saha (1993) (see also Abdellaoui et al. 2007), which can also be viewed as being in-between exponential and power utility. We briefly discuss the expo-power family in Section 6 below.

From the density function $U^{\prime}$ we obtain the cumulative distribution function $U$ as

$$
U(x)=1-\frac{x h^{-p}(x) \Gamma(p, p h(x))}{\lambda \Gamma(p)},
$$

where $\Gamma(p, h)=\int_{h}^{\infty} t^{p-1} \mathrm{e}^{-t} \mathrm{~d} t$ denotes the incomplete gamma function, and $\Gamma(p)=\Gamma(p, 0)$ is the (complete) gamma function; see Abramowitz and Stegun (1964, Chapter 5). This expression cannot be simplified unless $p$ is a positive integer, in which case we obtain

$$
\Gamma(p, p h(x))=\Gamma(p) \mathrm{e}^{-p h(x)} \sum_{k=0}^{p-1} \frac{p^{k} h^{k}(x)}{k !}
$$

and hence

$$
U(x)=1-\mathrm{e}^{-p h(x)} \sum_{k=0}^{p-1} \frac{p^{k} h^{k}(x)}{k !} .
$$

This specializes to exponential utility (2) when $p=1$, and to

$$
U(x)=1-\mathrm{e}^{-2 \sqrt{x / \lambda}}(1+2 \sqrt{x / \lambda})
$$

when $p=2$. Whether $p$ is a positive integer or not, one verifies that

$$
\operatorname{ARA}(x)=\frac{h(x)}{x}, \quad \operatorname{RRA}(x)=h(x)
$$

Like Pareto utility, gexpo utility is bounded from above and below (property P2), satisfies $\operatorname{RRA}(0)=0$, and has smooth derivatives at $x=0$. While $\mathrm{ARA}(0)=\infty$ for $p>1$, the property $\int_{0}^{\gamma} \operatorname{ARA}(x) \mathrm{d} x<\infty$ still holds for any $\gamma>0$ (P3). But unlike Pareto utility, gexpo utility does not behave power-like remote from the origin (property P4), since RRA increases without bound. Hence, for any $p \geq 1$, gexpo utility satisfies properties P1-P3, but not P4. Although this may be seen as a disadvantage of gexpo utility, there is empirical evidence that absolute risk tolerance is increasing and (strictly) concave in $x$ (Guiso and Paiella 2008), which would reject HARA (and Pareto) utility, but not gexpo utility. 


\section{Expo-power utility}

Somewhat related to gexpo utility is the well-known two-parameter expo-power family of utility functions, introduced by Saha (1993) and further developed by Saha et al. (1994) and Saha (1997), given by

$$
U(x)=1-\mathrm{e}^{-h(x)} \quad(p>0, \lambda>0),
$$

$\mathrm{x}$ where $h(x)$ is the same as in the previous section, that is, $h(x)=(x / \lambda)^{1 / p}$. The one-parameter special case of Abdellaoui et al. (2007) occurs when $\lambda=p^{-p}$ in which case $h(x)=p x^{1 / p}$.

The expo-power function is bounded between zero and one, and hence satisfies P2. It belongs to $\mathcal{U}$ (satisfies $\mathrm{P} 1$ ) if and only if $p \geq 1$. Since

$$
\operatorname{ARA}(x)=\frac{-1+p+h(x)}{p x}, \quad \operatorname{RRA}(x)=\frac{-1+p+h(x)}{p},
$$

we see that P3 holds if and only if $p=1$, in which case expo-power reduces to exponential utility; and that $\mathrm{P} 4$ does not hold for any $p>0$.

Expo-power utility is globally concave for $p \geq 1$, but convex-concave for $0<p<$ 1. This flexibility can be important for the purpose of fitting empirically individual choice data, in particular in a setting - not considered here-with both gain and loss inputs. We note in this respect that if $U(x)$ with $x \geq 0$ is gexpo (hence concave), then its generalization to negative inputs, $-U(-x)$ with $x<0$, is convex.

Comparing the well-known expo-power utility with gexpo utility, we see that, for $p>1$, both families satisfy properties $\mathrm{P} 1$ and $\mathrm{P} 2$, and that neither family satisfies $\mathrm{P} 4$. Also, absolute risk tolerance is increasing and concave for both families. But while gexpo satisfies P3, expo-power does not.

\section{Comparison of five utility functions}

In this section we shall compare, mostly graphically, the behavior of five members of the $\mathcal{U}$ class of utility functions:

exponential:

$$
U_{1}(x)=1-\mathrm{e}^{-x / \lambda_{1}}, \quad U_{1}^{\prime}(x)=\left(1 / \lambda_{1}\right) \mathrm{e}^{-x / \lambda_{1}},
$$

power:

$$
U_{2}(x)=\frac{x^{1-\alpha}-1}{1-\alpha}, \quad U_{2}^{\prime}(x)=x^{-\alpha},
$$

Pareto:

$$
U_{3}(x)=1-\frac{1}{\left(1+x / \lambda_{2}\right)^{k}}, \quad U_{3}^{\prime}(x)=\frac{k}{\lambda_{2}\left(1+x / \lambda_{2}\right)^{k+1}},
$$


gexpo:

$$
U_{4}(x)=1-\mathrm{e}^{-p_{1} h_{1}(x)} \sum_{k=0}^{p_{1}-1} \frac{p_{1}^{k} h_{1}^{k}(x)}{k !}, \quad U_{4}^{\prime}(x)=\frac{p_{1}^{p_{1}} \mathrm{e}^{-p_{1} h_{1}(x)}}{\lambda_{3} p_{1} !},
$$

expo-power:

$$
U_{5}(x)=1-\mathrm{e}^{-h_{2}(x)}, \quad U_{5}^{\prime}(x)=\frac{h_{2}(x) \mathrm{e}^{-h_{2}(x)}}{p_{2} x},
$$

where $h_{1}(x)=\left(x / \lambda_{3}\right)^{1 / p_{1}}, h_{2}(x)=\left(x / \lambda_{4}\right)^{1 / p_{2}}, \alpha>0, k>0, p_{1} \geq 2$ is an integer, $p_{2}>1$, and $\lambda_{i}>0(i=1, \ldots, 4)$. The ARA and RRA functions in the five cases are given by

$$
\begin{aligned}
& \operatorname{ARA}_{1}(x)=1 / \lambda_{1}, \quad \operatorname{ARA}_{2}(x)=\frac{\alpha}{x}, \quad \operatorname{ARA}_{3}(x)=\frac{k+1}{x+\lambda_{2}}, \\
& \operatorname{ARA}_{4}(x)=\frac{h_{1}(x)}{x}, \quad \operatorname{ARA}_{5}(x)=\frac{-1+p_{2}+h_{2}(x)}{p_{2} x},
\end{aligned}
$$

and

$$
\begin{aligned}
& \operatorname{RRA}_{1}(x)=x / \lambda_{1}, \quad \operatorname{RRA}_{2}(x)=\alpha, \quad \operatorname{RRA}_{3}(x)=\frac{(k+1) x}{x+\lambda_{2}}, \\
& \operatorname{RRA}_{4}(x)=h_{1}(x), \quad \operatorname{RRA}_{5}(x)=\frac{-1+p_{2}+h_{2}(x)}{p_{2}}
\end{aligned}
$$

In order to compare the five utility functions, we fix a point $x^{*}$ where we want the five functions to be 'close'. Without affecting the results, let us choose $x^{*}=0.08$. By 'close', we mean that RRA $\left(x^{*}\right)$ is the same for each of the five functions. If we choose $\alpha=2, k=1.5$, and $p_{1}=p_{2}=2$, then this condition implies $\lambda_{1}=0.04, \lambda_{2}=\lambda_{3}=$ 0.02 , and $\lambda_{4}=2 / 225$.

Figure 1 plots (normalized) marginal utility $\mathrm{MU}(x)=U^{\prime}(x) / U^{\prime}\left(x^{*}\right)$ for $0<x<$ 1.2 , and in the window zoomed in closer to the point $x^{*}=0.08$. Because of the normalizations, the five graphs are tangent at $x=x^{*}$, and the behavior of $\operatorname{MU}(x)$ near $x=x^{*}$ is dictated by the value of $U^{\prime \prime \prime}\left(x^{*}\right) / U^{\prime}\left(x^{*}\right)$, since

$$
\operatorname{MU}(x) \approx 1-\operatorname{ARA}\left(x^{*}\right)\left(x-x^{*}\right)+\frac{U^{\prime \prime \prime}\left(x^{*}\right)}{U^{\prime}\left(x^{*}\right)} \cdot \frac{\left(x-x^{*}\right)^{2}}{2} .
$$

We have, for values of $x$ close to $x^{*}$,

$$
\operatorname{MU}_{\text {power }}(x)>\operatorname{MU}_{\text {Pareto }}(x)>\operatorname{MU}_{\text {expo-power }}(x)>\operatorname{MU}_{\text {gexpo }}(x)>\operatorname{MU}_{\text {exp }}(x),
$$

while at $x=0, \operatorname{MU}_{\text {power }}(0)$ and $\mathrm{MU}_{\text {expo-power }}(0)$ are both infinity, and

$$
\operatorname{MU}_{\text {Pareto }}(0)=55.9, \quad \operatorname{MU}_{\text {gexpo }}(0)=54.6, \quad \operatorname{MU}_{\text {exp }}(0)=7.4 \text {. }
$$




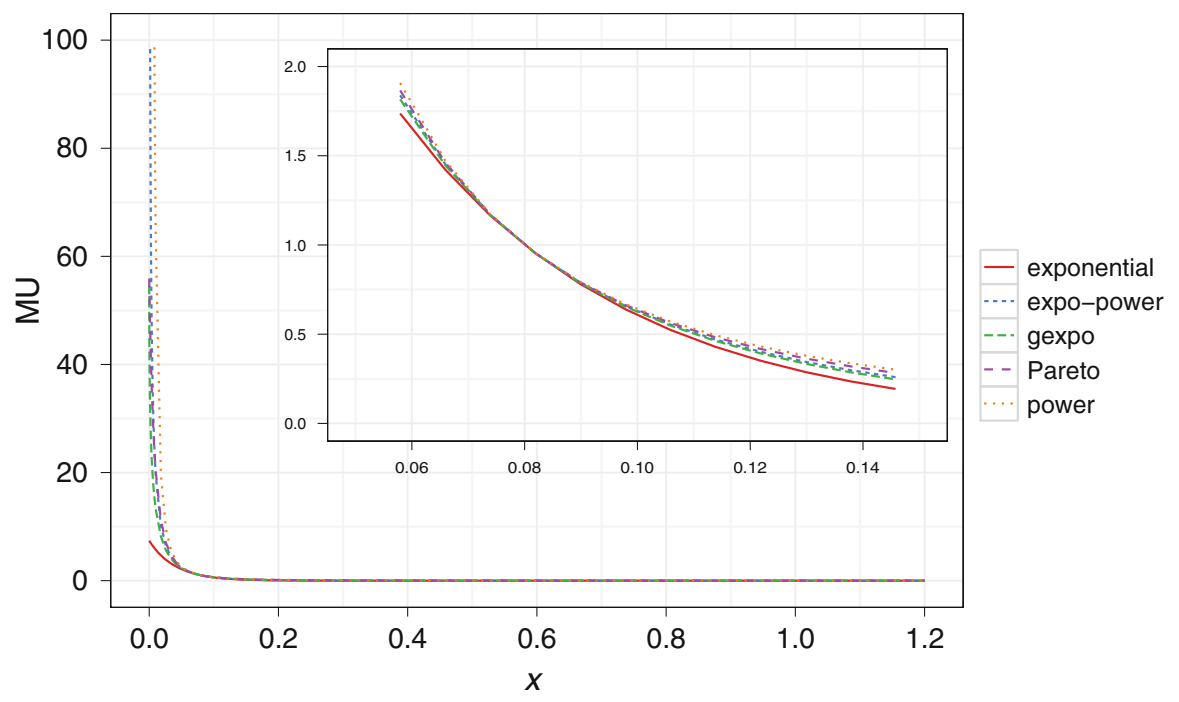

Fig. 1 Marginal utility (normalized) for five utility functions

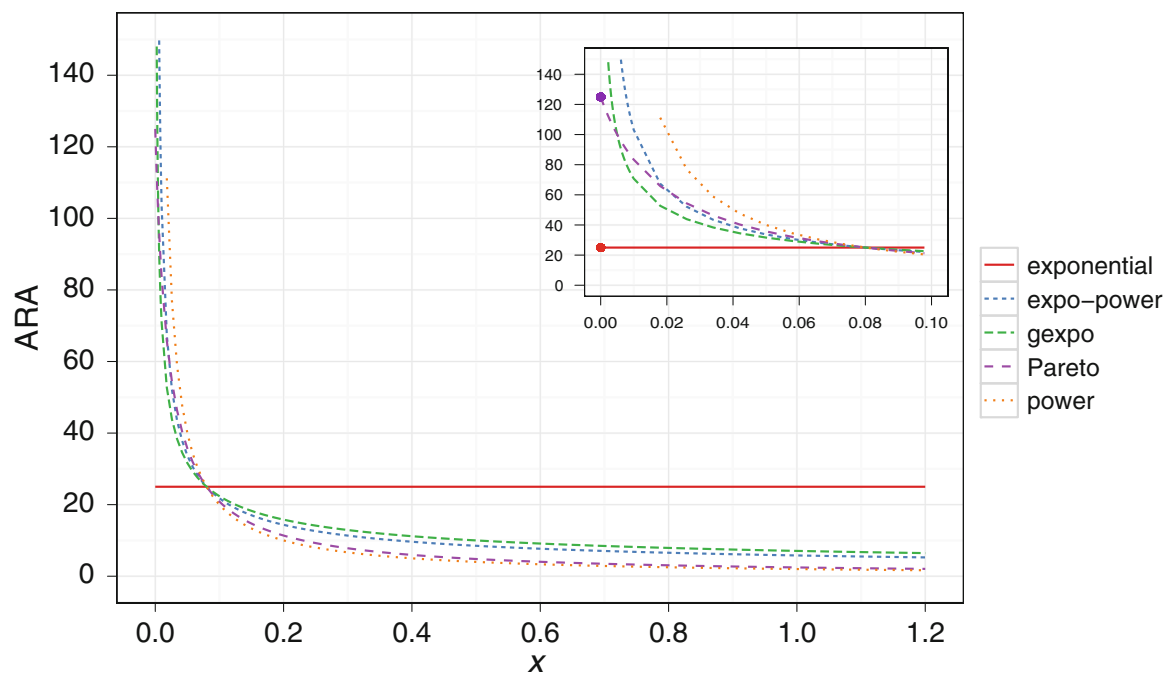

Fig. 2 Absolute risk aversion for five utility functions

Marginal utility is bounded from above and below except for the power and expopower functions.

Absolute risk aversion ARA is graphed in Fig. 2. We see that ARA(0) is finite for the Pareto and exponential families $\left(\operatorname{ARA} A_{\text {Pareto }}(0)=125\right.$ and $\left.\operatorname{ARA}_{\exp }(0)=25\right)$, but infinite for the power, expo-power, and gexpo families. While property P3 is not satisfied by the power and expo-power families, it is satisfied by the gexpo family since $\int_{0}^{\gamma} \operatorname{ARA}_{\text {gexpo }}(x) \mathrm{d} x<\infty$ for any $\gamma>0$. When $x$ is large, ARA Pareto is close to 


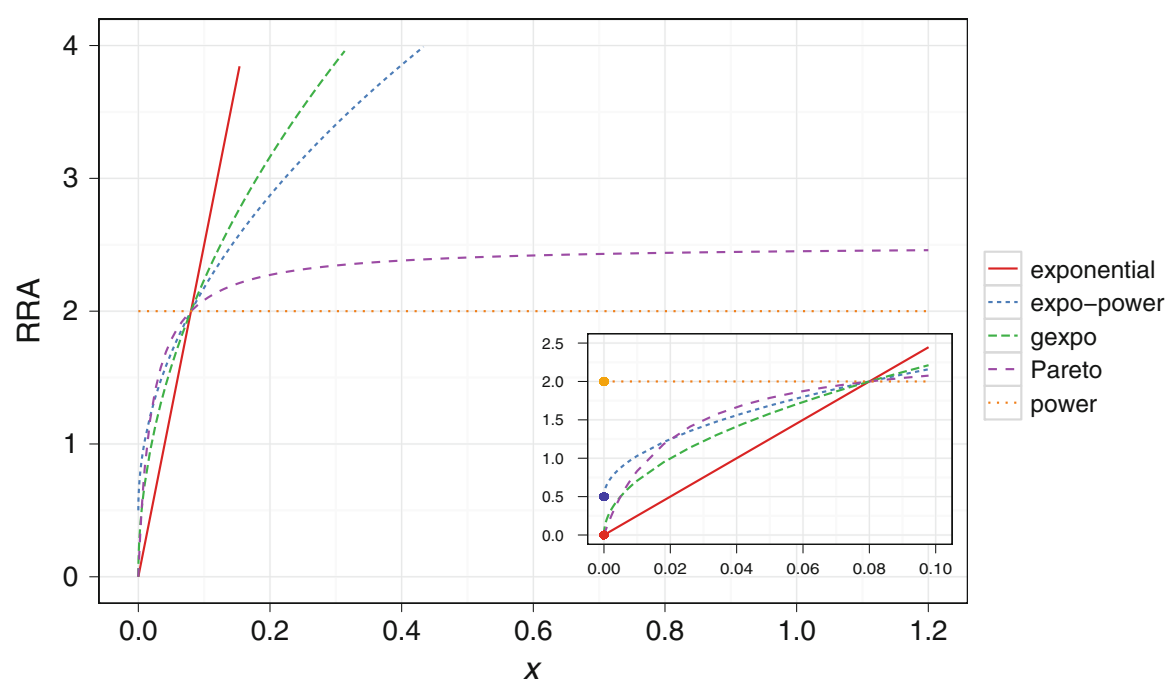

Fig. 3 Relative risk aversion for five utility functions

$\mathrm{ARA}_{\text {power }}$, but this is not the case when $x$ is small (close to 0). For small $x$, ARAPareto remains finite, just like $\mathrm{ARA}_{\text {expo }}$, but unlike power, expo-power, and gexpo.

In Fig. 3, we graph relative risk aversion RRA, arguably the most illustrative plot. It shows that $\mathrm{RRA}(0)=0$ except for power and expo-power utility, that Pareto, gexpo and expo-power lie in-between power and exponential, and that, when $x$ is large, gexpo and expo-power behave more like exponential, increasing without bound, while Pareto behaves more like power.

\section{Conclusions}

We have searched for a utility function satisfying four appealing properties, labeled P1-P4. We have been able to identify only one utility function that satisfies all four properties: Pareto utility. In the HARA class, Pareto utility is unique. In the Burr class of bounded utility functions, Pareto utility is also unique. Of course, other functions may exist satisfying the four properties, but it does not seem likely that they are as simple and parsimonious as Pareto utility.

Since Pareto utility is in the HARA class, its absolute risk tolerance is increasing and linear. In view of some empirical evidence, one may prefer absolute risk tolerance to be increasing and concave. Both expo-power and gexpo exhibit increasing and concave absolute risk tolerance, but there is a cost, namely that relative risk aversion increases without bound as $x$ becomes large. In a situation where infinite RRA is deemed less important than concave absolute risk tolerance, we find gexpo preferable over expo-power, because it satisfies our property P3, while expo-power does not.

The combination of our four properties is particularly relevant in catastrophic risk analysis, and Pareto utility is therefore well-suited for applications that require accounting for low-probability high-impact events. One example is economy-climate 
modeling where extreme climate changes are taken into account (Ikefuji et al. 2011). Another example is to account for rare adverse shocks in financial markets, in an attempt to resolve the equity premium puzzle of Mehra and Prescott (1985), along the lines of Barro $(2006,2009)$ who shows the potential of rare disasters in explaining this anomaly.

Acknowledgments We are grateful to Sjak Smulders and Peter Wakker for helpful discussions, and to the referee for constructive comments. This research was funded in part by the JSPS under grant C-22530177 (Ikefuji) and by the NWO under grant Vidi-2009 (Laeven). An earlier version of this article was circulated under the title 'Burr utility'.

Open Access This article is distributed under the terms of the Creative Commons Attribution License which permits any use, distribution, and reproduction in any medium, provided the original author(s) and the source are credited.

\section{References}

Abdellaoui, M., Barrios, C., \& Wakker, P. P. (2007). Reconciling introspective utility with revealed preference: Experimental arguments based on prospect theory. Journal of Econometrics, 138, 356378.

Abramowitz, M. \& Stegun, I. A. (Eds.). (1964). Handbook of mathematical functions with formulas, graphs, and mathematical tables. New York: Dover.

Arrow, K. J. (1971). Essays in the theory of risk bearing. Amsterdam: North-Holland.

Barro, R. J. (2006). Rare disasters and asset markets in the twentieth century. Quarterly Journal of Economics, 121, 823-866.

Barro, R. J. (2009). Rare disasters, asset prices, and welfare costs. American Economic Review, 99, 243264.

Binswanger, H. P. (1980). Attitude toward risk: Experimental measurement in rural India. American Journal of Agricultural Economics, 62, 395-407.

Box, G. E. C., \& Tiao, G. C. (1973). Bayesian inference in statistical analysis. Boston: Addison-Wesley.

Burr, I. W. (1942). Cumulative frequency functions. Annals of Mathematical Statistics, 13, $215-232$.

Burr, I. W., \& Cislak, P. J. (1968). On a general system of distributions: I. Its curve-shape characteristics. II The sample median. Journal of the American Statistical Association, 63, 627-635.

Castagnoli, E., \& LiCalzi, M. (1996). Expected utility without utility. Theory and Decision, 41, 281-301.

Chiappori, P.-A., \& Paiella, M. (2008). Relative risk aversion is constant: Evidence from panel data. Discussion paper no. 5/2008. Department of Economic Studies, University of Naples 'Parthenope', Naples.

Denuit, M., \& Eeckhoudt, L. (2010). Stronger measures of higher-order risk attitudes. Journal of Economic Theory, 145, 2027-2036.

de Finetti, B. (1952). Sulla preferibilità. Giornale Degli Economisti E Annali di Economia, 11, 685-709.

Eeckhoudt, L., \& Gollier, C. (1995). Risk: Evaluation, management and sharing. Hertfordshire: Harvester Wheatsheaf.

Friend, I., \& Blume, M. E. (1975). The demand for risky assets. American Economic Review, 65, 900-922.

Gerber, H. U. (1979). An introduction to mathematical risk theory. S.S. Huebner Foundation Monograph. Homewood, IL: Irwin.

Gollier, C. (2001). The economics of risk and time. Cambridge, MA: MIT Press.

Guiso, L., \& Paiella, M. (2008). Risk aversion, wealth, and background risk. Journal of the European Economic Association, 6, 1109-1150.

Harrison, G. W., List, J. A., \& Towe, C. (2007). Naturally occurring preferences and exogenous laboratory experiments: A case study of risk aversion. Econometrica, 75, 433-458.

Holt, C. A., \& Laury, S. K. (2002). Risk aversion and incentive effects. American Economic Review, 92, 1644-1655.

Ikefuji, M., Laeven, R. J. A., Magnus, J. R., \& Muris, C. (2011). Weitzman meets Nordhaus: Expected utility and catastrophic risk in a stochastic economy-climate model. Working paper, Tilburg University. 
Johnson, N. L., Kotz, S., \& Balakrishnan, N. (1995). Continuous univariate distributions (2nd ed., Vol. 2). New York: Wiley.

Köbberling, V., \& Wakker, P. P. (2005). An index of loss aversion. Journal of Economic Theory, 122, 119131.

Mehra, R., \& Prescott, E. C. (1985). The equity premium: A puzzle. Journal of Monetary Economics, 15, 145-161.

Merton, R. C. (1971). Optimum consumption and portfolio rules in a continuous-time model. Journal of Economic Theory, 3, 373-413.

Mossin, J. (1968). Optimal multiperiod portfolio policies. Journal of Business, 41, 215-229.

Post, T., van den Assem, M. J., Baltussen, G., \& Thaler, R. H. (2008). Deal or no deal? Decision making under risk in a large-payoff game show. American Economic Review, 98, 38-71.

Pratt, J. W. (1964). Risk aversion in the small and in the large. Econometrica, 32, 122-136.

Rabin, M. (2000). Risk aversion and expected-utility theory: A calibration theorem. Econometrica, $68,1281-1292$.

Saha, A. (1993). Expo-power utility: A flexible form for absolute and relative risk aversion. American Journal of Agricultural Economics, 75, 905-913.

Saha, A., Shumway, C. R., \& Talpaz, H. (1994). Joint estimation of risk preference structure and technology using expo-power utility. American Journal of Agricultural Economics, 76, 173-184.

Saha, A. (1997). Risk preference estimation in the nonlinear mean standard deviation approach. Economic Inquiry, 35, 770-782.

Stacy, E. W. (1962). A generalization of the gamma distribution. Annals of Mathematical Statistics, 33, 1187-1192.

Subbotin, M. Th. (1923). On the law of frequency of error. Mathematicheskii Sbornik, 31, $296-301$.

Wakker, P. P. (2008). Explaining the characteristics of the power (CRRA) utility family. Health Economics, 17, 1329-1344.

Yaari, M. (1969). Some remarks on measures of risk aversion and their uses. Journal of Economic Theory, 1, 315-329. 